\title{
Intraepithelial Lymphocyte Normal Cut-off Level in Celiac Disease: The Debate Continues
}

\author{
Aaron Lerner ${ }^{1,2, *}$, Torsten Matthias ${ }^{1}$ \\ ${ }^{1}$ AESKU.KIPP Institute, Wendelsheim, Germany \\ ${ }^{2}$ B. Rappaport School of Medicine, Technion-Israel Institute of Technology, Haifa, Israel \\ *Corresponding author: aaronlerner1948@gmail.com
}

Received November 10, 2015; Revised November 15, 2015; Accepted November 17, 2015

\begin{abstract}
The gold standard for diagnosis of celiac disease is positive serology confirmed by histological evidence of small intestinal biopsy damage as determined by the modified Marsh classification. Intraepithelial lymphocyte count has a pivotal importance in this grading criteria and the normal cut-off density has paramount importance in distinguishing between celiac disease and normal or false+ conditions. However its determination is problematic, complicated and far from being standardized. The localization along the villi or along the small bowel, the section thickness, the mode of detection and analysis, geoepidemiological influences, age dependency, associated infections or diseases and the strictness of the inclusions/exclusion criteria of the normal control group, all affect IEL density and normal cut-off levels. It is hoped that after standardization, the cut-off levels of IELs will better reflect the invivo reality to distinguish celiac disease from normal and associated conditions that affect intestinal IEL count.
\end{abstract}

Keywords: intraepithelial lymphocyte, celiac disease, intestine, diagnosis, children

Cite This Article: Aaron Lerner, and Torsten Matthias, "Intraepithelial Lymphocyte Normal Cut-off Level in Celiac Disease: The Debate Continues.” International Journal of Celiac Disease, vol. 4, no. 1 (2016): 4-6. doi: 10.12691/ijcd-4-1-1.

\section{Introduction}

In the routine daily practice, the gastroenterologist, the pediatrician and the family physician have to deal with extremely heterogeneous entities like atypical, silent, potential, latent or non-classical celiac disease (CD) forms $[1,2,3]$. In this complicated scenario, small bowel biopsy emerges as a pivotal tool along the diagnostic flow chart. Clinical reality is more complicated due to the multiproblematic and debatable subject of the best serological marker to screen for $C D$, which is an important early step to select the patient for an endoscopy [4]. Even the most frequently used autoantibody, IgA-tissue transglutaminase, is problematic and several competitors are challenging its primer ship [5,6,7,8]. At the end of the day, a robust diagnosis of $\mathrm{CD}$ is paramount for patient and clinician and the intestinal biopsy is a cast iron tool for a correct diagnosis, especially, when dealing with borderline/ gray zone, non-classical CD [1,9].

For the pathologist, the difficulties often lie in making the diagnosis at an early stage of $\mathrm{CD}$ progression. Milder or less prominent histological lesions are frequent in this multi-faced disease and increased intraepithelial lymphocytes (IEL) is a very early, or even the first histological presentation of the intestinal injury in CD. An increase in IEL count per 100 enterocytes along villi is a cardinal diagnostic feature of $\mathrm{CD}$, and it is the only abnormality found in Marsh type 1 lesion. However, it is not by itself sufficient for a definitive diagnosis of $C D$, as other conditions may present themselves in the same manner. Being an extremely important histological parameter, the cut-off number that distinguishes normal from CD intestinal injury, is the subject of the present editorial.

Siriweera EH et al [10], comparing IEL counts of adult $\mathrm{CD}$ patients to controls found "The upper limit of normal IEL/100EC (epithelial cells), mean IEL/100EC in CD and the villous tip IEL count in both the control and CD groups were considerably lower than those reported in other studies". At this stage, it is worthwhile to mention that the upper limit of normal IEL count/100EC, in the proximal small intestine has been reported to vary from 20-40. The official accepted and used number has long been considered to be 40 , as recommended by MarshOberhuber classification for CD [11]. It was determined by hematoxylin\&eosin staining on $7 \mu \mathrm{m}$-thick sections. It appears that defining a cut-off level of IEL is very problematic and controversial.

\section{Debatable Aspects in IEL Density in Celiac Disease and Controls}

The following are the methodological controversies concerning IEL count performance and reporting:

1. Topographical site along the villi/crypt length. Should the tips of villi or along villi length be counted? There is a tapering in IEL as we progress toward the villous apex [12]. 
2. Per how many EC should IEL be counted or reported? Per 20,100, 500 or 1000 EC [12].

3. The thickness of pathological sections impact IEL counts. Should it be a 3, 4 or $7 \mu \mathrm{m}$ thick section? Recent studies employing more thinly cut sections of $3 \mu \mathrm{m}$ and 4 $\mu \mathrm{m}$ have observed the upper limit of a normal count in the proximal small intestine to be lower [10].

4. Should H\&E staining or CD3 immunohistochemistry be employed? With the later staining IEL count is increased $[10,12]$.

5. Do traditional histological determinations or flow cytometry count? The advantages of flow cytometry are considerable compared to other user-dependent techniques, and results are obtained in a fast, sensitive, reproducible and objective semi-quantitative way just a few hours after taking the biopsy sample. It allows the analysis of a greater number of cells than immunohistochemistry does and yields a computerized record of the results [13]. IEL lymphogram, by flow cytometry, was suggested most recently to distinguish CD from healthy mucosa, obviating misinterpretation of minor histological changes, patchiness of the pathology and the concerns about the location and number of biopsies [14].

6. IEL count in non-celiac patients is significantly lower in the bulb than in the second part of the duodenum. These findings implicate that the site of biopsy should be taken into account when considering duodenal lymphocytosis. [15].

7. Differences among populations or ethnicity influence. The finger - like morphology of the villi may predominate in people residing in temperate areas, whereas those indigenous to more tropical climates often have the leaf like and ridge - like villous variants proximally and the finger - like variant distally [12]. The question arising is, do these morphological variants affect IEL count in those civilizations or topographical regions?

8. The level of IEL may be increased in regions overlaying intestinal lymphoid aggregates, areas that should be avoided when counting them.[16].

\section{Epidemiological, Environmental and Genetic Influences on IEL Counts}

Multiple genetic or environmental factors may affect intestinal IEL counts in normal and in gluten sensitive populations:

1. IEL number and subsets are age dependent [17]. Intestinal density of IELs in healthy subjects increases with age [18]. The counts were shown to be time dependent. During 11 years of follow up, an increased frequency of isolated increased IEL was detected [19].

2. Infections are a major influential factor, affecting IEL density. Viral enteritis, Helicobacter pylori, Giardiasis, bacterial overgrowth are some of them [12,16]. Of notice is the recent finding that IELs are not meaningfully influenced by Helicobacter pylori infection in children [20]. Of notice, Helicobacter pylori was not performed in Siriweera et al study [10].

3. As mentioned above, intestinal geomorphological variations exist in different societies [12]. Despite being an HLA-DQ dependent disease, CD was associated with a higher OR to IELs infiltrate $>25$, than to HLA-DQ2/8 (1640, 140, respectively) [21]. The importance of ruling out HLA-DQ2/8 carriers in the control groups used for the cut-off IEL normal level is important [18], not performed in Siriweera et al study [10]. Screening the genome wide associated studies performed on CD populations, multiple CD associated genes' functions are important in intestinal innate immunity. CTLA4, ICOSLG, IL2/IL21, PTPN22, SH2B3, STAT4, TAGAP and UBASH3A are some of them [22]. IEL-enterocyte cross talks are an integral part of the deregulated innate immunity in CD mucosa. It is logical to speculate that those CD susceptible genes influence IEL's regulation and impact their interepithelial density.

4. Numerous diseases and conditions are associated with duodenal interepithelial lymphocytosis (Table 1). Since CD and even "normal" controls may harbor comorbidity, one has to take into account their influence on IEL cut-off normal levels.

Table 1. Differential diagnosis of high intestinal IEL count*

\begin{tabular}{|l|l|}
\hline Disease category & Condition \\
\hline Anatomical & Blind loop syndrome \\
\hline Infectious & Bacterial overgrowth \\
\hline & Helicobacter pylori, Giardiasis, cryptosporid \\
\hline Allergy & To proteins/allergens \\
\hline Autoimmune diseases & Celiac disease, Crohn's disease, IBD, \\
\hline & $\begin{array}{l}\text { Dermatitis herpetiformis, SLE, Hashimoto } \\
\text { diabetes mellitus, Sjogren syndrome, autoim }\end{array}$ \\
\hline Drug induced & Non-steroidal anti-inflammatory \\
\hline Immunogenic & Graft-versus-host disease, common variable \\
\hline Nutritional & Kwashiorkor \\
\hline Cancerous & T cell lymphoma \\
\hline Physical & Radiation therapy \\
\hline Miscellaneous & Irritable bowel syndrome, peptic duodenitis, \\
Adapted from references [12,13,16,23]. \\
4. Quantity or Quality of IELs diagnostic \\
Importance
\end{tabular}

Even though the subject of the present editorial is on IEL quantitative density and cut-off levels, the more recent IEL cytometric studies highlight the importance of the IEL subsets' patterns, in CD diagnosis. The detectable abnormalities in the IEL compartment are an increase in the absolute and relative numbers of TcR- $\alpha \beta$ and TcR- $\delta$ IELs, even previous to the histological alterations and are more accurate than subepithelial deposits of anti-tissue transglutaminase IgA $[13,14]$. The analysis by flow cytometry of these IEL subsets (TcR- $\alpha \beta, T c R-\gamma \delta$ and $\mathrm{CD}^{-} \mathrm{CD} 103^{+}$) is a highly specific and sensitive immune- 
parameter in CD diagnosis, particularly in potential, latent, atypical or doubtful presentations [14]. It is foreseeable that in the future qualitative subsets will be more reliable than quantitative determination of IEL, in CD diagnosis.

\section{Conclusion}

The present manuscript highlights the importance of standardization of the methodology of intestinal IEL counting. Special emphasis should be put on a better definition of the normal groups. Due to their heterogeneity and despite their diagnostic importance in CD, IELs counts are far from being an absolute histological gold criteria for CD diagnosis.

\section{References}

[1] Lerner A, Neidhöfer S, Matthias T. Serological markers and/or intestinal biopsies in the case-finding of celiac disease. International Journal of Celiac Disease. 2015;3:53-55

[2] Lerner A, Amon-Levin N, Shapiro Y, Gilbert B, Reuter S, Lava L, Shoenfeld Y. The thrombophilia network of autoantibodies in celiac disease. BMJ Medicine, 2013, 11; 89-95.

[3] Lerner A. New Therapeutic Strategies for Celiac Disease. Autoimmune. Rev. 2010; 9:144-147.

[4] Lerner A. Serological Diagnosis of Celiac Disease -Moving Beyond the Tip of the Iceberg. International Journal of Celiac Disease. 2014; 2:64-66.

[5] Lerner A, Jeremias P, Matthias T. Outside of normal limits: false +/- anti TG2 autoantibodies. International Journal of Celiac Disease. 2015; 3:87-90

[6] Lerner A, Neidhöfer S, Matthias T. Transglutaminase 2 and anti transglutaminase 2 autoantibodies in celiac disease and beyond. Part B: Anti- Transglutaminase 2 autoantibodies: friends or enemies. Immunome Research , In Press, 2015.

[7] Lerner A, Neidhöfer S, Jeremias P, Matthias T. The diversities between the neo-epitope and the IgA- tissue transglutaminase autoantibodies in celiac disease. In: Autoantigens, autoantibodies, autoimmunity. Volume 10th , Eds: K Conrad, Chan EKL, Andrade LEC, Steiner G, Pruijn GJM, Y Shoenfeld. Page 220-226, e-pub, 2015.

[8] Jeremias P, Lerner A, Neidhöfer S, Matthias T. Industrial food additive microbial transglutaminase is immunogenic in children with celiac disease. 12th symposium on autoantibodies, 23-26.9.15, Dresden, Germany.
[9] Freeman HJ. Small intestinal mucosal biopsies for case-finding in celiac disease. International Journal of Celiac Disease. 2015;3:5052.

[10] Siriweera EH, Qi Z, Yong JLC. Validity of Intraepithelial Lymphocyte Count in the Diagnosis of Celiac Disease: A Histopathological Study. Internat J celiac Disease. 2015; 3: 156158.

[11] Antonioli D. Coeliac disease :A progress report. Mod Pathol. 2003;6;342-346.

[12] Dickson BC, Streutker CJ, Chetty R. Coeliac disease: an update for pathologists. J Clin Pathol. 2006;59:1008-1016.

[13] Fernández-Bañares F, Carrasco A, García-Puig R wet al. Intestinal intraepithelial lymphocyte cytometric pattern is more accurate than subepithelial deposits of anti-tissue transglutaminase IgA for the diagnosis of celiac disease in lymphocytic enteritis. PLoS One. 2014;9:e101249.

[14] De Andrés A, Camarero C, Roy G. Distal duodenum versus duodenal bulb: intraepithelial lymphocytes have something to say in celiac disease diagnosis. Dig Dis Sci. 2015;60:1004-1009.

[15] Bednarska O, Ignatova S, Dahle C, Ström M. Intraepithelial lymphocyte distribution differs between the bulb and the second part of duodenum. BMC Gastroenterol. 2013;13:111-115.

[16] Hammer ST, Greenson JK. The clinical significance of duodenal lymphocytosis with normal villus architecture. Arch Pathol Lab Med. 2013;137:1216-1219.

[17] Camarero C, Leon F, Sanchez L, Asensio A, Roy G. Age-related variation of intraepithelial lymphocytes subsets in normal human duodenal mucosa. Dig Dis Sci. 2007;52:685-691.

[18] Pellegrino S, Villanacci V, Sansotta N, et al. Redefining the intraepithelial lymphocytes threshold to diagnose gluten sensitivity in patients with architecturally normal duodenal histology. Aliment Pharmacol Ther. 2011;33:697-706.

[19] Shmidt E, Smyrk TC, Boswell CL, Enders FT, Oxentenko AS Increasing duodenal intraepithelial lymphocytosis found at upper endoscopy: time trends and associations. Gastrointest Endosc. 2014;80:105-111.

[20] Guz-Mark A, Zevit N, Morgenstern S, Shamir R. Duodenal intraepithelial lymphocytosis is common in children without coeliac disease, and is not meaningfully influenced by Helicobacter pylori infection. Aliment Pharmacol Ther. 2014;39:1314-1320

[21] Losurdo G, Piscitelli D, Giangaspero A, et al. Evolution of nonspecific duodenal lymphocytosis over 2 years of follow-up. World J Gastroenterol. 2015;21:7545-7552.

[22] Lerner A, Matthias T. Rheumatoid arthritis-celiac disease relationship: joints get that gut feeling. Autoimm Rev. 2015:14:1038-1047.

[23] Chang F, Mahadeva U, Deere H. Pathological and clinical significance of increased intraepithelial lymphocytes (IELs) in small bowel mucosa. APMIS. 2005;113:385-99. 